\title{
COMMUTATIVITY OF UNBOUNDED REPRESENTATIONS
}

\author{
SCHÔICHI ÔTA \\ (Communicated by Palle E. Jorgensen)
}

\begin{abstract}
We introduce the notion of commutativity for unbounded representations of a *-algebra, and we study integrable or selfadjoint extensions, with a condition of the commutativity, of a representation.
\end{abstract}

\section{INTRODUCTION}

Unbounded representations of a $*$-algebra have been studied with close relation to quantum field theory $[1,9,11,15]$. In this paper, introducing the notions of strong commutativity and weak commutativity for representations, we study extensions of unbounded representations under some commutativity conditions. An integrable representation plays an important role in making deeper analyses on representations (see [11;13, Chapters 9 and 10]), and so we shall be concerned with integrable extensions of a representation. On the other hand, there are some arguments on commuting selfadjoint extensions of unbounded operators in a Hilbert space (for example, [6, 14]) and on commuting derivations in $C^{*}$-algebras [10]. Motivated by them, we show in $\S 3$ that two commuting integrable extensions of a representation coincide, and we also show that for a closed $*$-representation $\pi$ there is no proper selfadjoint extension of $\pi$ that strongly commutes with $\pi$.

In case of commutative algebras, it is shown in [13, Proposition 9.1.12] that every integrable extension of a representation can be obtained by its induced extension and, as we will see in Corollary 4 , any integrable extension of a representation $\pi$ weakly commutes with $\pi$. In $\S 4$ we discuss the integrability of the induced extension commuting with a representation.

\section{Preliminaries}

We begin with definitions of unbounded representations. Let $\mathfrak{A}$ be a *-algebra with unit $e$. Let $\pi$ be a linear mapping of $\mathfrak{A}$ into all closable linear operators on a common dense subspace $\mathscr{D}(\pi)$ of a Hilbert space $\mathscr{H}$. If $\pi$ satisfies that $\pi(e)=I$, the identity operator on $\mathscr{l}$, and $\mathscr{D}(\pi)$ is invariant under each $\pi(x)$ and $\pi(x) \pi(y) \eta=\pi(x y) \eta$ for all $x, y \in \mathfrak{A}$ and $\eta \in \mathscr{D}(\pi)$,

Received by the editors July 22, 1991.

1991 Mathematics Subject Classification. Primary 47D40, 46K40.

Key words and phrases. Unbounded representation, strong commutativity, commuting extension, induced representation. 
then $\pi$ is said to be a representation of $\mathfrak{A}$ on $\mathscr{H}$. If the domain $\mathscr{D}(\pi)$ is complete with respect to the induced topology given by the family of seminorms $\{\|\pi(x) \xi\|: x \in \mathfrak{A}\}, \pi$ is called closed. If a representation $\pi$ satisfies

$$
(\pi(x) \xi, \eta)=\left(\xi, \pi\left(x^{*}\right) \eta\right)
$$

for all $x \in \mathfrak{A}$ and $\xi, \eta \in \mathscr{D}(\pi)$, then $\pi$ is said to be a $*$-representation.

We now define a closed representation derived from $\pi$ by

$$
\begin{aligned}
& \mathscr{D}\left(\pi^{*}\right)=\bigcap_{x \in \mathfrak{A}} \mathscr{D}\left(\pi(x)^{*}\right), \\
& \pi^{*}(x)=\left.\pi\left(x^{*}\right)^{*}\right|_{\mathscr{D}\left(\pi^{*}\right)} \quad\left(\text { the restriction of } \pi\left(x^{*}\right)^{*} \text { to } \mathscr{D}\left(\pi^{*}\right)\right) .
\end{aligned}
$$

If $\pi=\pi^{*}$, we call $\pi$ selfadjoint. A closed $*$-representation $\pi$ is called integrable (or standard in terms of [11]) if $\overline{\pi\left(a^{*}\right)}=\pi(a)^{*}$ for all $a \in \mathfrak{A}$. Clearly, an integrable representation is selfadjoint. We should note that a closed *-representation $\pi$ is integrable if and only if $\pi(a)$ is essentially selfadjoint for all $a=a^{*} \in \mathfrak{A}$.

We next recall definitions of weak and strong commutants of representations. For a representation $\pi$ of $\mathfrak{A}$ on $\mathscr{H}$, the weak commutant $\mathscr{C}^{w}(\pi)$ is defined by

$$
\mathscr{C}^{w}(\pi)=\left\{T \in \mathscr{B}(\mathscr{H}): T \pi(x) \subset \pi^{*}(x) T \text { for all } x \in \mathfrak{A}\right\},
$$

and the strong commutant $\mathscr{C}^{s}(\pi)$ is defined by

$$
\mathscr{C}^{s}(\pi)=\{T \in \mathscr{B}(\mathscr{H}): T \pi(x) \subset \pi(x) T \text { for all } x \in \mathfrak{A}\} .
$$

Here $\mathscr{B}(\mathscr{H})$ denotes the algebra of all bounded linear operators on $\mathscr{H}$. If $\pi$ is selfadjoint, then $\mathscr{C}^{w}(\pi)$ is a von Neumann algebra with $\mathscr{C}^{w}(\pi)=\mathscr{C}^{s}(\pi)$. For further details on unbounded representations, we refer to [13].

Definition 1. Let $\pi_{1}$ and $\pi_{2}$ be $*$-representations of $\mathfrak{A}$ on a Hilbert space $\mathscr{H}$. We say that $\pi_{1}$ strongly commutes with $\pi_{2}$ if the commutant of $\mathscr{C}^{s}\left(\pi_{1}\right)$ is contained in $\mathscr{C}^{s}\left(\pi_{2}\right) ; \mathscr{C}^{s}\left(\pi_{1}\right)^{\prime} \subset \mathscr{C}^{s}\left(\pi_{2}\right)$. Analogously, we say that $\pi_{1}$ weakly commutes with $\pi_{2}$ if $\mathscr{C}^{s}\left(\pi_{1}\right)^{\prime} \subset \mathscr{C}^{w}\left(\pi_{2}\right)$. Here, for a subset $\mathscr{M}$ of $\mathscr{B}(\mathscr{H}), \mathscr{M}^{\prime}$ denotes the usual commutant of $\mathscr{M}$.

The commutativity of representations is not symmetric; that is, if $\pi_{1}$ strongly commutes with $\pi_{2}$, then $\pi_{2}$ does not commute strongly with $\pi_{1}$ in general. Moreover, the weak commutativity does not always imply the strong commutativity. Clearly, the above two notions coincide for the class of selfadjoint representations and, for such representations $\pi_{1}$ and $\pi_{2}$, if $\pi_{1}$ strongly commutes with $\pi_{2}$ then $\pi_{2}$ strongly commutes with $\pi_{1}$.

Example 2. Let $\mathfrak{A}=\mathbb{C}[a]$ be a free commutative algebra with unit $e$ generated by one hermitian element $a$. Suppose $\pi_{1}$ and $\pi_{2}$ are selfadjoint representations of $\mathfrak{A}$ on a Hilbert space $\mathscr{H}$. Then by [5], each $\pi_{i}(a)(i=1,2)$ is essentially selfadjoint and $\mathscr{D}\left(\pi_{i}\right)=\bigcap_{n=1}^{\infty} \mathscr{D}\left({\overline{\pi_{i}(a)}}^{n}\right)$. It follows from the spectral theory that

$$
\mathscr{C}^{s}\left(\pi_{i}\right)=\mathscr{C}^{w}\left(\pi_{i}\right)=\left\{e^{i \overline{\pi_{i}(a)}}: t \in \mathbb{R}\right\}^{\prime} \quad(i=1,2) .
$$

Hence $\pi_{1}$ strongly commutes with $\pi_{2}$ if and only if selfadjoint operators $\overline{\pi_{1}(a)}$ and $\overline{\pi_{2}(a)}$ strongly commute. 
Proposition 3. Let $\pi$ be a selfadjoint representation of a *-algebra $\mathfrak{A}$ on a Hilbert space. If $\pi$ strongly commutes with itself then $\pi$ is integrable. Conversely, if $\pi$ is integrable and, in addition, $\mathfrak{A}$ is abelian, then $\pi$ is strongly commutes with itself.

Proof. Since $\pi$ is selfadjoint and $\mathscr{C}^{s}(\pi)^{\prime} \subset \mathscr{C}^{s}(\pi)$ by the definition, $\mathscr{C}^{s}(\pi)^{\prime}$ is an abelian von Neumann algebra. For each $a=a^{*} \in \mathfrak{A}$, the closed symmetric operator $\overline{\pi(a)}$ is affiliated with $\mathscr{C}^{s}(\pi)^{\prime}$, and so $\overline{\pi(a)}$ is selfadjoint (see $\left.[8, \S 5.6]\right)$. This means that $\pi$ is integrable. Conversely, suppose $\pi$ is integrable. Since each $\pi(a)\left(a=a^{*} \in \mathfrak{A}\right)$ is essentially selfadjoint, it follows from the definition of the commutant and [13, Corollary 8.2.8] that the von Neumann algebra $\mathscr{C}^{s}(\pi)^{\prime}$ is generated by the unitary operators $e^{i \overline{\pi(a)}}$ with all hermitian $a$ in $\mathfrak{A}$. Therefore if, in addition, $\mathfrak{A}$ is abelian, then it follows from [13, Proposition 7.1.3 and Theorem 9.1.2] that $\mathscr{C}^{s}(\pi)^{\prime}$ is abelian. Thus $\pi$ is strongly commutes with itself.

For *-representations $\pi$ and $\rho$ of a $*$-algebra $\mathfrak{A}$ on a Hilbert space $\mathscr{H}$, the relation $\pi \subset \rho$ means that $\mathscr{D}(\pi) \subset \mathscr{D}(\rho)$ and $\pi(a) \eta=\rho(a) \eta$ for all $a \in \mathfrak{A}$ and all $\eta \in \mathscr{D}(\pi)$, and if this relation holds, then $\rho$ is said to be an extension of $\pi$.

Corollary 4. Suppose $\mathfrak{A}$ is an abelian $*$-algebra and $\pi$ is a *-representation of $\mathfrak{A}$ on a Hilbert space. Then any integrable extension of $\pi$ weakly commutes with $\pi$.

Proof. Let $\rho$ be an integrable extension of $\pi$. It follows that $\mathscr{C}^{w}(\rho) \subset \mathscr{C}^{w}(\pi)$. The proposition follows from Proposition 3.

\section{Commuting extensions}

Let $B$ be a closed symmetric operator with equal deficiency indices on a Hilbert space $\mathscr{H}$ and $B_{0}$ be a selfadjoint extension of $B$. Let $\mathfrak{A}$ be a $*$ algebra $\mathbb{C}[a]$ of Example 2. Define two *-representation $\pi$ and $\rho$ of $\mathfrak{A}$ on $\mathscr{H}$ by

$$
\begin{aligned}
\mathscr{D}(\pi) & =\bigcap_{n=1}^{\infty} \mathscr{D}\left(B^{n}\right), \\
\pi(a) & \left.=\left.B\right|_{\mathscr{D}(\pi)} \quad \text { the restriction of } B \text { to } \mathscr{D}(\pi)\right)
\end{aligned}
$$

and

$$
\begin{aligned}
\mathscr{D}(\rho) & =\bigcap_{n=1}^{\infty} \mathscr{D}\left(B_{0}^{n}\right), \\
\rho(a) & \left.=\left.B_{0}\right|_{\mathscr{D}(\rho)} \quad \text { (the restriction of } B_{0} \text { to } \mathscr{D}(\rho)\right) .
\end{aligned}
$$

By Corollary $4, \rho$ weakly commutes with $\pi$ and so the inequality $e^{i t B_{0}} B \subset$ $B^{*} e^{i t B_{0}}$ holds. Especially, if $e^{i t B_{0}} B \subset B e^{i t B_{0}}(t \in \mathbb{R})$, then it follows from the spectral theory that $B=B_{0}$; namely, $\pi=\rho$. We note that this condition is equivalent to: $\rho$ strongly commutes with $\pi$. In general, we have

Theorem 5. Let $\pi$ be a $*$-representation of a *-algebra $\mathfrak{A}$ on a Hilbert space $\not{H}$. Suppose closed $*$-representations $\rho_{1}$ and $\rho_{2}$ are extensions of $\pi$. If $\rho_{2}$ is integrable and $\rho_{2}$ strongly commutes with $\rho_{1}$, then $\rho_{1}$ and $\rho_{2}$ coincide. 
Proof. Suppose $\rho_{2}$ is integrable. Take $a=a^{*}$ in $\mathfrak{A}$. Then the closed symmetric operator $\overline{\rho_{2}(a)}$ is selfadjoint. In the rest of the proof, we write $T_{0}, T_{1}$, and $T_{2}$ for the closures $\overline{\pi(a)}, \overline{\rho_{1}(a)}$, and $\overline{\rho_{2}(a)}$, respectively. It follows from the spectral theory that the resolvent $\left(i-T_{2}\right)^{-1}$ of $T_{2}$ at $i$ strongly commutes with $T_{2}$, where $i$ denotes $\sqrt{-1}$. Hence,

$$
\left(i-T_{2}\right)^{-1} T_{0} \subset\left(i-T_{2}\right)^{-1} T_{2} \subset T_{2}\left(i-T_{2}\right)^{-1}=-I+i\left(i-T_{2}\right)^{-1},
$$

so that $\left(i-T_{2}\right)^{-1} T_{0}$ is continuous.

Since $T\left(i-T_{2}\right) \subset\left(i-T_{2}\right) T$ for all $T \in \mathscr{C}^{s}\left(\rho_{2}\right)$, it follows that $\left(i-T_{2}\right)^{-1}$ belongs to $\mathscr{C}^{s}\left(\rho_{2}\right)^{\prime}$. Since $\rho_{2}$ strongly commutes with $\rho_{1},\left(i-T_{2}\right)^{-1}$ belongs to $\mathscr{C}^{s}\left(\rho_{1}\right)$. Thus

$$
\left(i-T_{2}\right)^{-1} T_{0} \subset\left(i-T_{2}\right)^{-1} T_{1} \subset T_{1}\left(i-T_{2}\right)^{-1} .
$$

Since $T_{1}\left(i-T_{2}\right)^{-1}$ is closed, it follows that $T_{1}\left(i-T_{2}\right)^{-1}$ is everywhere defined and is equal to the continuous extension of $\left(i-T_{2}\right)^{-1} T_{0}$. This means that

$$
T_{1}\left(i-T_{2}\right)^{-1}=T_{2}\left(i-T_{2}\right)^{-1},
$$

so that $T_{1}=T_{2}$. Hence $\rho_{1}(a)$ is essentially selfadjoint for each $a=a^{*}$ in $\mathfrak{A}$. This is equivalent that $\rho_{1}$ is integrable. Since $\overline{\rho_{1}(a)}=\overline{\rho_{2}(a)}$ for all $a=a^{*}$ in $\mathfrak{A}$, it follows that $\rho_{1}=\rho_{2}$.

Remark 1. By Corollary 4, Theorem 5 does not hold in general in the case that $\rho_{2}$ weakly, but not strongly, commutes with $\rho_{1}$. Let $\pi$ be a $*$-representation of $\mathfrak{A}$ on $\mathscr{H}$. For $a=a^{*} \in \mathfrak{A}$, let $V_{\pi(a)}$ be the Cayley transformation of the closed symmetric operator $\overline{\pi(a)}$;

$$
V_{\pi(a)}=(\overline{\pi(a)}-i)(\overline{\pi(a)}+i)^{-1} .
$$

Then, as usual, $V_{\pi(a)}$ is extended to the partial isometry on $\mathscr{H}$ that is zero on the deficiency space $\operatorname{ker}\left(\pi(a)^{*}-i\right)$ and it is also denoted by $V_{\pi(a)}$.

We remark that $\pi(a)$ is essentially selfadjoint if $V_{\pi(a)} V_{\pi(a)}^{*} \in \mathscr{C}^{w}(\pi)$ and $V_{\pi(a)}^{*} V_{\pi(a)} \in \mathscr{C}^{w}(\pi)[4]$.

Theorem 6. Let $\pi$ be a closed *-representation of a *-algebra $\mathfrak{A}$ on a Hilbert space $\mathscr{H}$. If $\pi$ has a selfadjoint extension that strongly commutes with $\pi$, then $\pi$ is integrable and consequently has no proper extensions.

Proof. Suppose $\rho$ is a selfadjoint representation of $\mathfrak{A}$ such that $\rho$ strongly commutes with $\pi$ and $\rho \supset \pi$. Then we have only to show that $\rho=\pi$. Take $a=a^{*}$ in $\mathfrak{A}$ and $B \in \mathscr{C}^{w}(\rho)^{\prime}$. Since $\mathscr{C}^{w}(\rho)^{\prime}=\mathscr{C}^{s}(\rho)^{\prime} \subset \mathscr{C}^{s}(\pi), B$ and $B^{*}$ belong to $\mathscr{C}^{s}(\pi)$. Since $B$ strongly commutes with the closed symmetric operator $\overline{\pi(a)}$, it follows that

$$
B V_{\pi(a)} \xi=V_{\pi(a)} B \xi
$$

for all $\xi \in \mathscr{R}(\overline{\pi(a)}+i)$, the range of $\overline{\pi(a)}+i$. On the other hand, $B$ also strongly commutes with $\pi(a)^{*}$. For $\xi \in \mathscr{R}(\overline{\pi(a)}+i)^{\perp}=\operatorname{ker}\left(\pi(a)^{*}-i\right)$, we have $\pi(a)^{*} B \xi=B \pi(a)^{*} \xi=i B \xi$. Hence $B \xi \in \mathscr{R}(\overline{\pi(a)}+i)^{\perp}$, and so the partial isometry $V_{\pi(a)}$ and $B$ commute. Therefore $V_{\pi(a)} \in \mathscr{C}^{w}(\rho)$. By the relation $\rho \supset \pi, \mathscr{C}^{w}(\rho) \subset \mathscr{C}^{w}(\pi)$. Since $\mathscr{C}^{w}(\rho)$ is a $*$-algebra, $V_{\pi(a)} V_{\pi(a)}^{*}$ and $V_{\pi(a)}^{*} V_{\pi(a)}$ belong to $\mathscr{C}^{w}(\pi)$. It follows from Remark 1 that $\pi(a)$ is essentially selfadjoint. This means that $\pi$ is integrable and $\pi=\rho$. 
Remark 2. Let $\pi_{1}$ and $\pi_{2}$ be *-representations of $\mathfrak{A}$ on $\mathscr{H}$. Suppose $\pi_{2}$ is an extension of $\pi_{1}$ and a *-representation $\rho$ weakly commutes with $\pi_{2}$. Then it is easily seen that $\rho$ weakly commutes with $\pi_{1}$. Conversely, for a *-representation $\pi$ of $\mathfrak{A}$ on $\mathscr{H}$, suppose a selfadjoint (or integrable) representation $\rho$ of $\mathfrak{A}$ on $\mathscr{H}$ strongly commutes with $\pi$. Then it is natural to ask whether there exists a selfadjoint (or integrable) extension $\pi_{0}$ of $\pi$ in $\mathscr{H}$ such that $\rho$ strongly (or weakly) commutes with $\pi_{0}$. However, we can easily construct a counterexample by using [14, the mention just before the proof of Theorem 3.1].

\section{INDUCED REPRESENTATIONS}

In this section we consider the commutativity of induced extensions related to $[4 ; 13, \S \S 8.5$ and 9.1$]$. Let $\mathscr{M}$ be a subset of $\mathscr{C}^{w}(\pi)$. We define a representation $\pi^{0}$ of $\mathfrak{A}$ with the domain $\mathscr{D}\left(\pi^{0}\right)$ consisting of the linear span of $\mathscr{M} \mathscr{D}(\pi)$ by

$$
\pi^{0}(x)\left(\sum_{k} C_{k} \xi_{k}\right)=\sum_{k} C_{k} \pi(x) \xi_{k}
$$

for $x \in \mathfrak{A}$ and $\sum_{k} C_{k} \xi_{k} \in \mathscr{D}\left(\pi^{0}\right)$. We write $\pi_{\mathscr{M}}$ for the closure of $\pi^{0}$, which is called the induced representation of $\pi$ determined by $\mathscr{M}$.

If $\mathscr{M}$ is a subalgebra containing the identity operator $I$, then $\pi$ is a *-representation and $\mathscr{M}$ is contained in the strong commutant of the induced representation $\pi_{\mathscr{M}}$ of $\mathscr{M}$; that is, $\mathscr{M} \subseteq \mathscr{C}^{s}\left(\pi_{\mathscr{M}}\right)$. If, in addition, $\mathscr{M}$ is a *-subalgebra, then $\pi_{\mathscr{M}}$ is an extension of $\pi$ on $\mathscr{H}$ as a $*$-representation with $\pi \subset \pi_{\mathscr{M}} \subset \pi^{*}$. We call $\pi_{\mathscr{M}}$ the induced extension of $\pi$.

Lemma 7. Let $\pi$ and $\rho$ be *-representations of $a *$-algebra $\mathfrak{A}$. If $\rho$ is an extension of $\pi$, then $\pi_{\mathscr{C}^{s}(\rho)}$ is well defined and is an extension of $\pi$ as $a$ *-representation satisfying $\pi \subset \pi_{\mathscr{C}^{s}(\rho)} \subset \rho$.

Proof. Since $\rho \supset \pi$, we have $\mathscr{C}^{s}(\rho) \subset \mathscr{C}^{w}(\rho) \subset \mathscr{C}^{w}(\pi)$. Hence, $\pi_{\mathscr{C} s}(\rho)$ is well defined as an induced representation of $\pi$ and clearly $\pi \subset \pi_{\mathscr{C} s}(\rho)$. Since $\mathscr{C}^{s}(\rho) \mathscr{D}(\pi) \subset \mathscr{D}(\pi)$, it follows that $\pi_{\mathscr{C}^{s}(\rho)}(x) T \eta=T \pi(x) \eta=T \rho(x) \eta=$ $\rho(x) T \eta$, for all $T \in \mathscr{C}^{s}(\rho)$ and $\eta \in \mathscr{D}(\pi)$. Thus, $\pi_{\mathscr{C} s}(\rho) \subset \rho$, and so $\pi_{\mathscr{C} s(\rho)}$ is a *-representation.

Proposition 8. Suppose $\pi$ is a *-representation of a *-algebra $\mathfrak{A}$ on a Hilbert space $\mathscr{H}$ and $\rho$ is a selfadjoint extension of $\pi$, and suppose $\rho$ weakly commutes with $\pi$. If one of $\rho, \pi_{\mathscr{C} s}(\rho)$, and $\pi_{\mathscr{C}^{s}(\rho)^{\prime}}$ is integrable, then all of them coincide.

Proof. Since $\rho$ weakly commutes with $\pi$, it follows that $\pi_{\mathscr{C} s}(\rho)^{\prime}$ is well defined as an induced representation and $\pi \subset \pi_{\mathscr{C}^{s}}(\rho)^{\prime}$. Since $\rho$ is selfadjoint, $\mathscr{C}^{s}(\rho)$ is a von Neumann algebra on $\mathscr{H}$ and so $\pi_{\mathscr{C} s}(\rho)$ is an extension of $\pi$ as a *-representation. Moreover, $\mathscr{C}^{s}(\rho)^{\prime} \subset \mathscr{C}^{s}\left(\pi_{\mathscr{C}} s(\rho)^{\prime}\right)$; that is, $\rho$ strongly commutes with $\pi_{\mathscr{C} s}(\rho)^{\prime}$. And we have

$$
\mathscr{C}^{s}\left(\pi_{\mathscr{C}}(\rho)^{\prime}\right)^{\prime} \subset \mathscr{C}^{s}(\rho)^{\prime \prime}=\mathscr{C}^{s}(\rho) \subset \mathscr{C}^{s}\left(\pi_{\mathscr{C} s}(\rho)\right)
$$

This means that $\pi_{\mathscr{C} s}(\rho)^{\prime}$ strongly commutes with $\pi_{\mathscr{C} s}(\rho)$.

We first assume that $\rho$ is integrable. Then $\rho=\pi_{\mathscr{C}^{s}(\rho)^{\prime}}$ by Theorem 5 , so that $\rho$ strongly commutes with $\pi_{\mathscr{C} s}(\rho)$. Hence $\rho=\pi_{\mathscr{C}}(\rho)$. 
We next assume that $\pi_{\mathscr{C} s(\rho)}$ is integrable. We have only to show that $\rho$ is integrable. Since a selfadjoint extension is unique, $\rho=\pi_{\mathscr{C} s}(\rho)$. Thus $\rho$ is integrable.

Lastly we assume that $\pi_{\mathscr{C} s}(\rho)^{\prime}$ is integrable. Since $\pi_{\mathscr{C}_{s}(\rho)^{\prime}}$ strongly commutes with $\pi_{\mathscr{C}_{s}(\rho)}$, it follows from Theorem 5 that $\pi_{\mathscr{C}_{s}(\rho)}$ is integrable. From the above argument, the proposition follows.

Remark 3. Under the same assumption as in Proposition 8 except for the selfadjointness of $\rho$, we can show that if one of $\rho$ and $\pi_{\mathscr{C}_{s}(\rho)}$ is integrable then $\rho=\pi_{\mathscr{C}^{s}(\rho)}=\pi_{\mathscr{C} s}(\rho)^{\prime}$, by a slight modification of the proof above.

Proposition 9. Let $\pi$ be a selfadjoint representation of a *-algebra $\mathfrak{A}$ on a Hilbert space. Suppose there is a subalgebra $\mathscr{M}$ contained in $\mathscr{C}^{w}(\pi)$ such that the induced representation $\pi_{\mathscr{K}}$ weakly commutes with $\pi$. Then $\pi$ is integrable. Proof. Define $\mathscr{N}=\mathscr{C}^{w}\left(\pi_{\mathscr{M}}\right)$. Since $\pi_{\mathscr{M}}$ weakly commutes with $\pi$ and $\pi_{\mathscr{M}} \supset$ $\pi, \mathscr{N}^{\prime} \subset \mathscr{C}^{s}\left(\pi_{\mathscr{M}}\right)^{\prime} \subset \mathscr{C}^{w}(\pi)$ and $\mathcal{N} \subset \mathscr{C}^{w}(\pi)$. Hence $\mathscr{C}^{w}(\pi)^{\prime \prime} \supset \mathscr{C}^{w}(\pi) \supset$ $\mathscr{N}^{\prime} \supset \mathscr{C}^{w}(\pi)^{\prime}$. Thus, for each $a=a^{*} \in \mathfrak{A}$, the closed symmetric operator $\overline{\pi(a)}$ is affiliated with the abelian von Neumann algebra $\mathscr{C}^{w}(\pi)^{\prime}$, and so $\overline{\pi(a)}$ is selfadjoint. This means that $\pi$ is integrable.

\section{REFERENCES}

1. H. J. Borchers, Algebraic aspects of Wightman field theory in statistical mechanics and field theory lectures, Halsted Press, New York, 1972.

2. H. J. Borchers and J. Yngvason, On the algebra of field operators. The weak commutant and integral decompositions of states, Comm. Math. Phys. 42 (1975), 231-252.

3. S. Gudder and W. Scruggs, Unbounded representations of *-algebras, Pacific J. Math. 20 (1977), 369-382.

4. A. Inoue, H. Kurose, and S. Ôta, Extensions of unbounded representations, Math. Nachr. (to appear).

5. A. Inoue and K. Takesue, Selfadjoint representations of polynomial algebras, Trans. Amer. Math. Soc. 280 (1983), 393-400.

6. P. E. T. Jørgensen, Selfadjoint extension operators commuting with an algebra, Math. Z. 169 (1979), 41-62.

7. - Operators and representation theory, North-Holland, Amsterdam, 1988.

8. R. V. Kadison and J. R. Ringrose, Fundamentals of the theory of operator algebras, Vol. 1, Academic Press, New York, 1983.

9. G. Lassner, Topological algebras of operators, Rep. Math. Phys. 3 (1972), 279-293.

10. S. Ôta, Commutants of unbounded derivations in $C^{*}$-algebras, J. Reine Angew. Math. 347 (1984), 21-32.

11. R. T. Powers, Self-adjoint algebras of unbounded operators, Comm. Math. Phys. 21 (1971), 85-124.

12. M. Reed and B. Simon, Methods of modern mathematical physics, Vol. 1, Academic Press, New York, 1972.

13. K. Schmüdgen, Unbounded operator algebras and representation theory, Akademie-Verlag, Berlin, 1988.

14. S. P. Slinker, On commuting self-adjoint extensions of unbounded operators, Indiana Univ. Math. J. 27 (1978), 629-636.

15. A. N. Vasilev, Theory of representations of a topological (non-Banach) involutory algebra, Theoret. Math. Phys. 2 (1970), 113-123.

Department of Mathematics, Kyushu Institute of Design, Fukuoka, 815 Japan

E-mail address: ota@kyushu-id.ac.jp 